\title{
Pulse Oximeter Manufacturing \& Wireless Telemetry for Ventilation Oxygen Support
}

\author{
Ali İhsan Bülbül *1, Serdar Küçük ${ }^{2}$ \\ Accepted $3^{\text {rd }}$ September 2016
}

\begin{abstract}
Pulse Oximeter devices are widely used as a non-invasive method for instant monitoring of blood oxygen saturation and heart rate. In this paper, a wireless microcontroller based pulse oximeter is proposed to measure the oxygen delivered to the patient via the oxygen flowmeter. In the first step, the signals received from reusable $\mathrm{SpO}_{2}$ sensor (finger probe) are processed by a microcontroller to determine the blood oxygen saturation and heart rate. Depending on the current blood oxygen saturation value, wireless signals are sent to the non-invasive ventilation flow meter vacuum regulator to deliver the necessary oxygen into the patient. Oxygen supplied to the patient is automatically controlled according to the oxygen saturation change.
\end{abstract}

Keywords: Pulse Oximeter, Oxygen saturation, Ventilation, Oxygen flow meter, Oxygen regulator, Circuit design, Wireless control.

\section{Introduction}

Oxygen is the common drug to be used in the care of patients who present with medical emergencies. Currently, ambulance and emergency department teams are likely to give oxygen to all breathless a large number of patients [1].

The amount of oxygen saturated hemoglobin in arterial blood is expressed with oxygen saturation [2]. Formerly, the most common method of assessing oxygenation was the use of arterial blood gases [3]. This is painful for patients and has the serious complications of vascular injury or occlusion and infection. The methods of applying also pose the risk of needle stick injury to staff [4].

Today, pulse oximetry is a safe and simple method of assessing oxygenation [2]. At the same time, they are cheap and easy to carry devices. Pulse oximeter is a measuring device as peripheral arterial oxygen saturation in the blood non-invasively. Pulse oximeter shown in Figure 1, can be monitored from bedside monitors as used alone. Pulse oximeter is required intraoperatively monitors. During patient monitoring using pulse oximeters the early detection of untoward events is the most important, especially as it may contribute to the prevention of hypoxic insults [5].

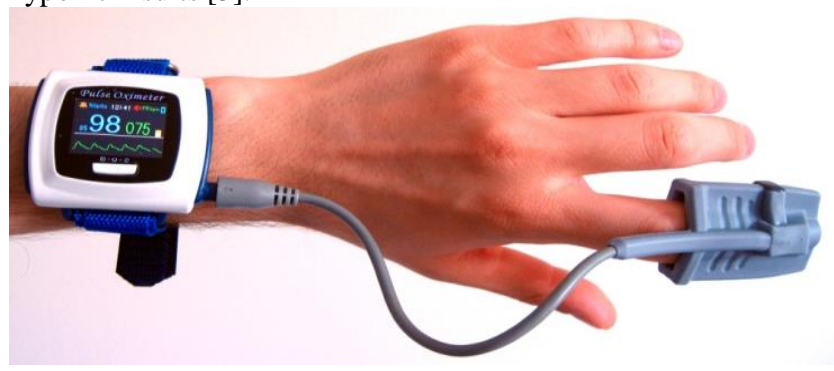

Figure 1. Pulse oximeter.

\footnotetext{
${ }^{1}$ Biomedical Engineering, Technology Faculty, Kocaeli University, Kocaeli /Turkey

* Corresponding Author: Email: aliihsanbulbul@yahoo.com Note: This paper has been presented at the $3^{\text {rd }}$ International Conference on Advanced Technology \& Sciences (ICAT'16) held in Konya (Turkey), September 01-03, 2016.
}

Pulse oximetry is a routine device used in surgery, intensive care units and operating rooms, due to its cheapness. It is a noninvasive device and easy to be used. [6].

Oxygen flowmeter illustrated in Figure 2 is used to give the oxygen to the patient through the humidifier, nose nozzle and mask. Vacuum regulator allows patients to adjust the vacuum level. It has the manometer to see visually the pressure. Vacuum regulator is set manually as shown in figure 2 .

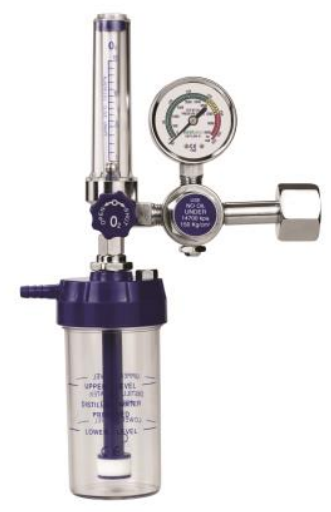

Figure 2. Oxygen Flowmeter, Vacuum regulator.

The patient may need to be supported by mechanical ventilation when the respiratory system fails [7]. Mechanical ventilation is common form of life support in the intensive care unit (ICU) [8]. Although mechanical ventilation is one of the ultimate lifesupporting technologies, in recent years, there has been renewed interest in the injury that it can cause. The concept that high airway pressures during positive pressure ventilation can cause gross injury manifest has been well known and investigated for a long time [9]. It was found that an average of $40 \%$ of the patients died in the process of finalizing mechanical ventilation. It was found that also a high overall mortality in the intensive care unit [10].

In the literature, there have been several studies on pulse oximetry. Although the small number of wireless applications, studies involving with oxygen flow meter have not been a part of literature. Some of the studies in the literature on pulse oximetry and wireless communications are as follows: Watthanawisuth and 
his team (2010) worked on the monitoring of pulse oximetry with wireless wearable system [11]. Qing Cai, Jinming Sun, Ling Xia and Xingqun Zhao (2011), have created a wireless pulse oximeter sensor using a wrist strap [12]. Turban and Niwayama (2011) have worked on reducing the power consumption of wireless pulse oximeter [13]. Rekha Chandra, Safer and Srividya (2015) worked on the development and miniaturization of wireless pulse oximeter [14]. Render, Ayaz and Dalkılıç (2014) worked based on the Arduino Healthduino wireless Mobile Health Monitoring System, cloud-based database, doctors and patients mobile applications [15]. In the study by using RF technology. Adochie and colleagues wireless pulse oximeter is designed. Also it has been monitored with WiFi or GSM / GPRS technology [16].

The studies mentioned above do not both measure oxygen saturation and supply oxygen automatically to the patients. The aim of this study is to design a system that measure first the oxygen saturation and provide the required oxygen to the patients via a wireless and oxygen flowmeter system. For this purpose, a microcontroller is used to measure the blood oxygen saturation. A wireless signal including the measured blood oxygen saturation data is sent to the non-invasive ventilation flow meter vacuum regulator to deliver the required oxygen into the patient. This process is repeated as the doctor's recommendation continues. Thus, according to symptomatic status of the patient, mechanical ventilation causing pre-intervention to acute respiratory failure is no mere required. By using proposed device in this study, $\mathrm{O}_{2}$ saturation can be precisely arranged and delivered to the patients. Microcontroller programming with proper protocols based on the different patient stories and different diseases can prevent the problems caused by human influences.

\section{Pulse Oximetry}

Respiratory system are specialized to allow gas exchange between ambient air and blood. The 97\% part of oxygen composes of chemical compounds with the hemoglobin in the red blood cell and rest of $3 \%$ composes of dissolved in the fluid plasma and cells. Oxygen saturation is defined as the ratio of hemoglobin to total hemoglobin bound to oxygen in the blood.

The $\mathrm{O}_{2}$ saturation is close to $100 \%$ under normal conditions. The $\mathrm{O}_{2}$ saturation between $97 \%$ and $100 \%$ represents good gas exchange within the person. Hypoxia is mentioned above when $\mathrm{SpO}_{2}$ level falls below $90 \%$ and may require respiratory support. Identifying hypoxia conditions in an early stage is important. Thus an early intervention becomes possible and therapy starts immediately.

According to the blood gas analysis method, 'arterial oxygen saturation' is represented by the symbol $\mathrm{SaO}_{2}$ in the literature. This parameter corresponds to the oximetry method 'arterial oxyhemoglobin concentration' (represented by the measured $\mathrm{SpO}_{2}$ symbol). The main difference between $\mathrm{SaO}_{2}$ and $\mathrm{SpO}_{2}$ parameters is that $\mathrm{SpO}_{2}$ presents the amount of oxygen bound to the hemoglobin molecule found while $\mathrm{SaO}_{2}$ denotes the total amount of oxygen in the arteries [17].

Oximeters are devices that determine the oxygen concentration of various species of $\mathrm{Hb}$ [18].

\subsection{Principles of Pulse Oximeter}

Light is transmitted, absorbed, or reflected when it passes through matter. The relative absorption or reflection of light at different wavelengths is used in several monitoring devices to estimate the concentrations of dissolved substances. This type of measurement is called as spectrophotometry and is based on the Beer-Lambert Law. According to the Beer-Lambert Law, if a known intensity of light illuminates a chamber of known dimensions, then the concentration of a dissolved substance can be determined if the incident and transmitted light intensity is measured [18].

The pulse oximetry is based on two physical principles. The first one is the light absorbance of oxygenated hemoglobin and the second one is the absorbance at different wavelengths with pulsatile (AC) component [19]. Pulse oximeter is based on the principles to absorb light different level by oxygenated hemoglobin with reduced hemoglobin [14].

The form of $\mathrm{Hb}$ is important issue. In the adult blood usually contains four species of $\mathrm{Hb}: \mathrm{HbO}_{2}$, reduced $\mathrm{Hb}$, methemoglobin (metHb), and carboxyhemoglobin $(\mathrm{COHb})$. Each of $\mathrm{Hb}$ species has a different light absorption profile. The different absorption constants for each $\mathrm{Hb}$ species over a range of light from red to infrared are shown in Figure 3 [18].

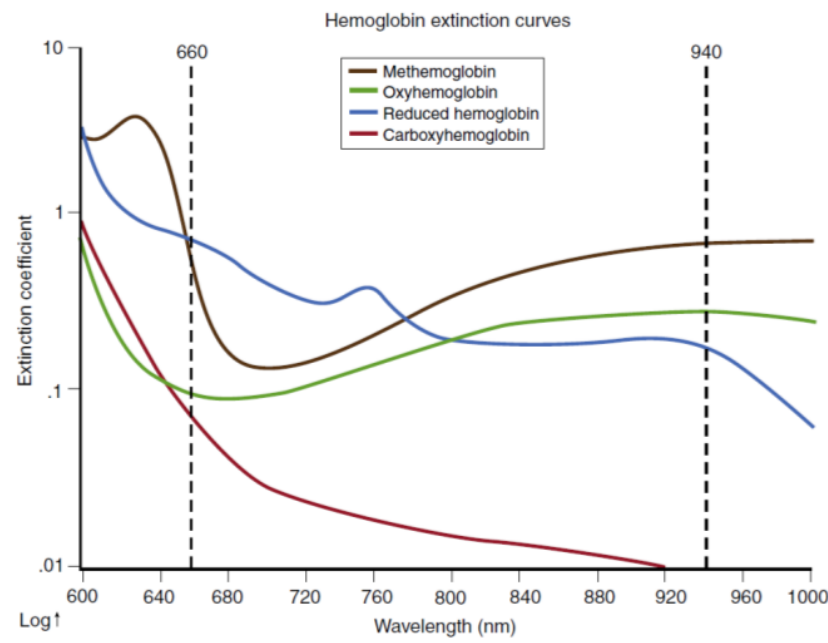

Figure 3: Hemoglobin extinction curves.

Pulse oximetry uses the wavelengths of $660 \mathrm{~nm}$ and $940 \mathrm{~nm}$ because these wavelengths are available in semiconductors.

The oximeters measure red and infrared light transmitted through and reflected by a tissue bed. There are several technical problems to accurate estimation of $\mathrm{SaO}_{2}$ by this method. For example, many light absorbers other than arterial $\mathrm{Hb}$ are in the transmitted light path (e.g., skin, soft tissue, venous). A group of absorbers in a typical sample of living tissue is shown in Figure 4 [18].

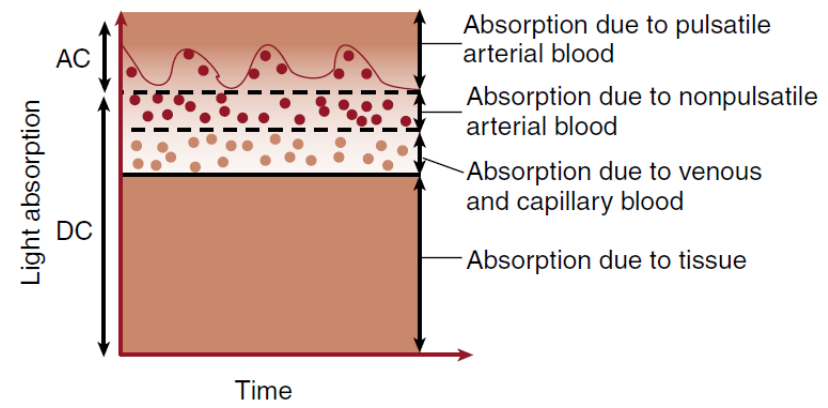

Figure 4: Pulse oximetry signals.

The AC component represents absorption of light by the pulsating arterial blood at the top of the figure. The DC (baseline) component represents absorption of light by the tissue layer. The pulse oximeters mostly use only two wavelengths of light, typically $660 \mathrm{~nm}$ (red light) and $940 \mathrm{~nm}$ (infrared light). The pulse oximeter first determines the fluctuating or AC component of absorbance at two wavelengths. Secondly it divides this value 
by the DC component to obtain the pulse-added absorbance which is independent of the incident light intensity. Thus, the oximeter then calculates the ratio $(\mathrm{R})$ of the two pulse-added absorbance (one for each wavelength) [19]:

$R=\frac{A C_{660} / D C_{660}}{A C_{940} / D C_{940}}$

Pulse oximetry is calibrated using the calculated value of R. An example of a pulse oximeter calibration curve is shown in Figure 5 [22].

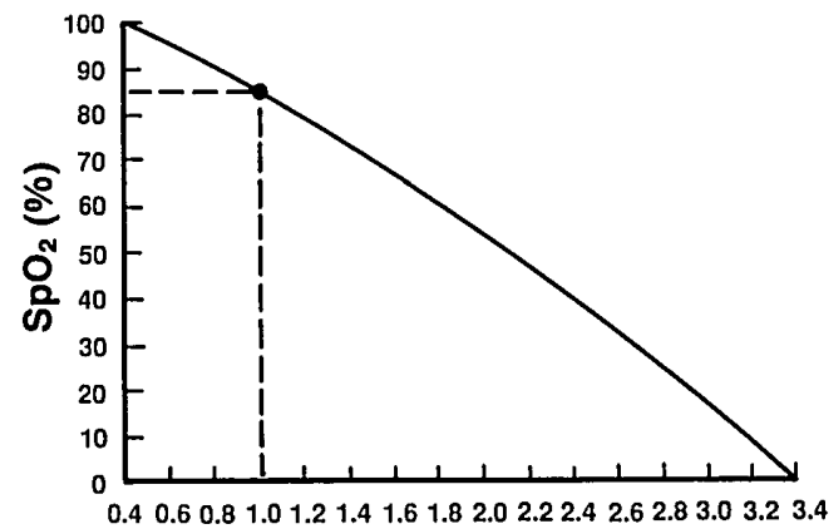

$$
R=\frac{A C_{660} / D C_{660}}{A C_{940} / D C_{940}}
$$

Figure 5: Typical pulse oximeter calibration curve.

The curve used in all commercial pulse oximeters are based on experimental studies in healthy human volunteers. Although each manufacturer is different, these curves are similar [18].

\section{2. $\mathrm{SpO}_{2}$ Probe}

Biosensors are important tools in food safety, diagnostics, medical monitoring and biological warfare agent's detection systems. Pulse oximeter probe is a sensor that detects oxygen.

The pulse oximeter probes are used for the purpose of evaluation of the patient's oxygen saturation; it is the best perfused on fingers, toes, nose area, ear lobes.

In adult and pediatric size probes are manufactured in accordance with multiple and disposable use (Fig. 6).

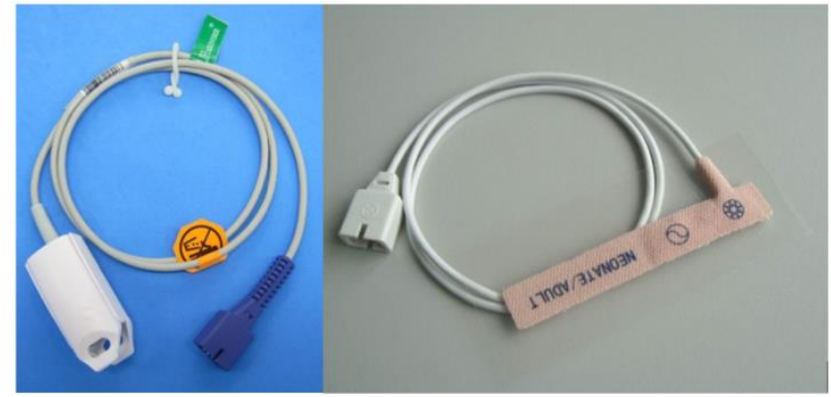

Figure 6: Reusable and disposable $\mathrm{SpO}_{2}$ probes.

The attenuation characteristics of light passing through the fingertip consist of three components: tissue loss, and (vein) the weakening of the artery (artery) attenuation. Tissue attenuation and veins attenuation have a constant characteristic. Arteries (arterial) blood flow in fingertip causes light attenuation variation. The reason is the change in the oxygen saturation of the blood pumped to the body blow to the heart of each pulse. Heart rate (pulse) signal is superimposed on the fixed component which is weakening. Thus, arterial oxygen saturation can be calculated by subtracting from the total attenuation of the attenuation constant and vein tissue attenuation components [17].

The $\mathrm{SpO}_{2}$ value can be read incorrectly in some situations, environmental effects, and technical. These conditions are:

- Very bright light in the operating room (such as more lighting).

- Abnormalities in value of carboxyhemoglobin and methemoglobin.

- Electrocautery device can be prevented by transportation of signal to diode when it running.

- It may be affected by light emitted from the surgical light in the hall.

- $\mathrm{SpO}_{2}$ value can be measured or read incorrectly when the patient's fingernails very long, fingernails with diamonds, henna, dyed and so on.

\section{Pulse Oximeter Manufacturing And Wireless Telemetry For Ventilation Oxygen Support}

In this study, a telemetry control system is proposed to support oxygen to the patients based to the data measured by pulse oximeter. The schematic diagram of proposed wireless telemetry system for ventilation oxygen support with pulse oximeter is shown in Figure 7.

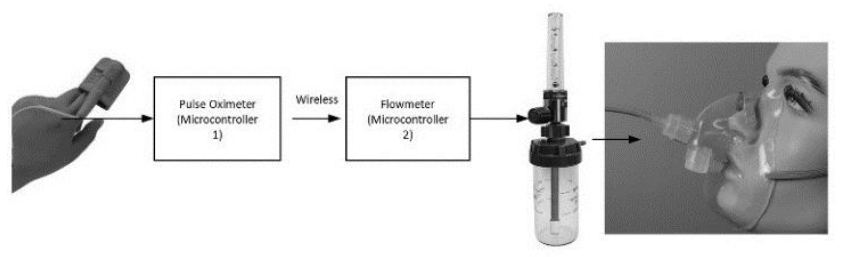

Figure 7: Wireless telemetry for ventilation oxygen support with pulse oximeter.

\subsection{Pulse Oximeter Manufacturing}

The pulse oximeter block diagram in Figure 7 is illustrated in Figure 8 in detail. Pulse oximeter is proposed to design using microcontroller.

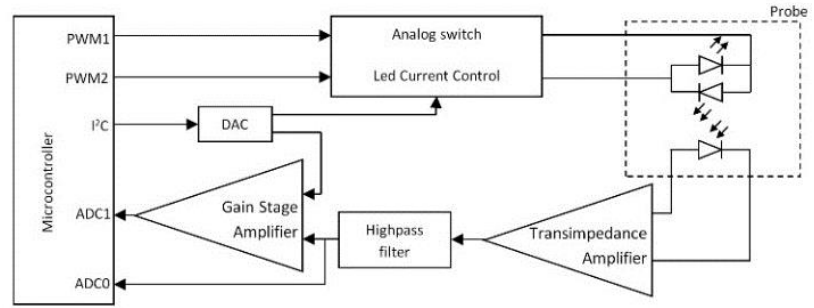

Figure 8: Block diagram of pulse oximeter.

The probe consists of a photodiode and two LEDs including red and infrared LEDs. The $\mathrm{SpO}_{2}$ sensor LEDs are connected as shown in Figure 9. 


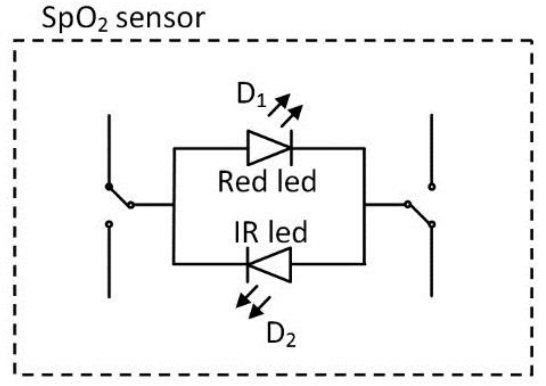

Figure 9: $\mathrm{SpO}_{2}$ sensor leds.

The LEDs are controlled by the microcontroller PWM signal. However, the microcontroller PWM output cannot provide enough power to drive properly led. LEDs are forced to give enough energy by the led driver circuit. A finger probe measures the oxygen saturation via pulse oximeter probe as analog signal. Since the only one photodetector for the two LED signals, the signals cannot be received at the receiver at the same time, thus requiring switching of signals. Double the switching drive integrated, red and infrared LEDs with two signals from the microcontroller does turn on and off. The timing diagram to drive the LEDs is shown in Figure 10.

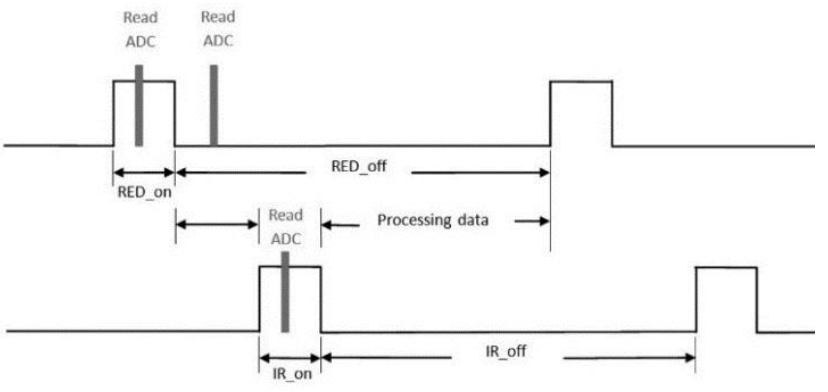

Figure 10: Timing diagram.

Photodiode generates an analog current depending on the light absorption. This current must turn into voltage. That transformation is performed in analog signal conditioning circuit. The analog signal conditioning circuit is illustrated as in Figure 11 .

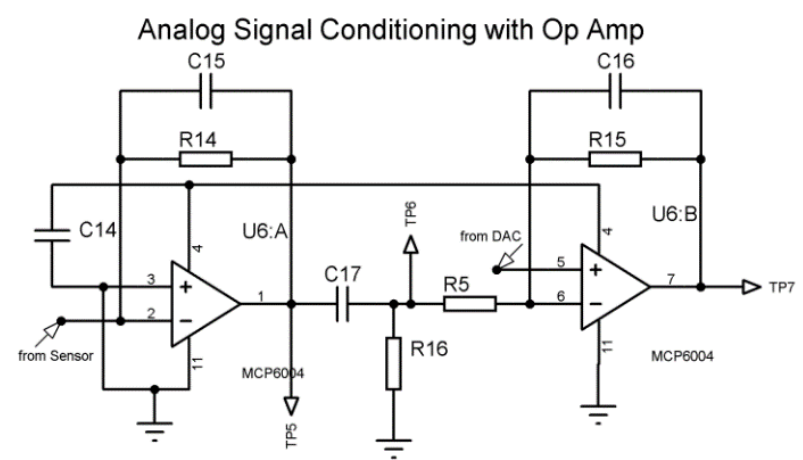

Figure 11: Analog signal conditioning circuit.

The $\mathrm{SpO}_{2}$ is calculated when the signal is applied to AnalogDigital Converter (ADC) module in microcontrollers. Signal conditioning circuit consists of three stages. First stage is amplifier (transimpedance amplifier), which takes a few microamps current by photodiode and turning into a few millivolts. Transimpedance amplifier is an amplifier as well as current- voltage converter. The signal passes by High Pass Filter (HPF). Therefore background light interference is reduced. High Pass Filter output is upgraded with second stage gain amplifier.

There are two filter applications. The first one is a passive RC filter illustrated within a circle in Figure 11. The second one is in the microcontroller as software. The digital filter is called FIR. Microcontroller include Digital Signal Processing (DSP) unit. DSP performs digital Finite Impulse Response (FIR) that is the filtered data. FIR is a software filter. The filtered data is used to calculate the pulse amplitude. The pulse amplitude is used to calculate $\mathrm{SpO}_{2}$ and heart rate.

Finally microcontroller sends the computed data about blood oxygen saturation to the second microcontroller via wireless unit.

\subsection{Wireless Oxygen Flowmeter Control}

Flowmeter manually operate in general. In this study it is operated automatic control with a motor and motor driver circuit. It uses pulse oximeter wireless data for automatic control.

\subsubsection{Oxygen Supply And Equipment}

Oxygen is supplied for patients from two sources, including central system and $\mathrm{O}_{2}$ tubing. Flowmeter (oxygen flow measuring equipment) and humidifier (humidification oxygen canister) is located in the patients unit. It is shown in Figure 2.

The pressure in the oxygen tube is very high to provide oxygen to the patient. The pressure to be used medically appropriate level (40-70 psi-pounds) must be reduced. Therefore, regulator is used in order to regulate the pressure. Regulator is an arranger by display.

Flowmeter adjusts the flow rate per minute of oxygen delivered to the patient. Humidifier is a chamber used depending flowmeter. It allows the moisture of the oxygen from the oxygen source.

In patients with respiratory; nasal cannula, simple face mask, partial mask recycled, recyclable mask, venturi mask, nebulizer mask and oxygen header methods are used. In patients without respiratory mask balloon system (Baggins valve- mask) is used [14].

\subsubsection{Motor Control Circuit}

In this application, a motor is mounted on the flowmeter. The data is taken from a pulse oximeter with wireless communication module.

The motor control system is shown in figure 12. This system provides motor control with wireless data from pulse oximeter. It is controlled motor speed and left-right rotation by implementation of the appropriate voltage values.

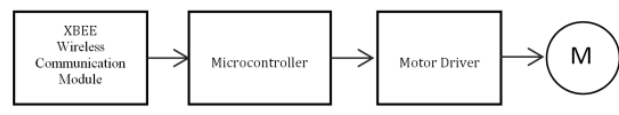

Figure 12: Motor control system block diagram

The data from the pulse oximeter is named as A0 and A1. Equation 2 is used for to determine motor speed.

motor $=K_{1} \cdot A_{1}+K_{0} \cdot A_{0}$

$\mathrm{K} 0$ and $\mathrm{K} 1$ expressions in the equation 2 are coefficients used to determine the applied voltage to the motor according to the received value.

If the pulse oximeter measurement $\mathrm{SpO}_{2}$ value falls under 95\%, then the motor works. If it falls under $90 \%$ then the oxygen transferred to the patient is increased. If the measurement value rise above $95 \%$, then rotation reversed valve is closed. 
Motor situation and $\mathrm{SpO}_{2}$ values can be changed easily in the microcontroller. Thus working system can be programmable according to doctor's protocol.

\section{Conclusion}

Developments in electronics and sensor technology yields several advantages for biomedical applications. For example, analog and digital devices for bio-signal treatment can easily be employed by microcontrollers.

Biosensors and pulse oximeter probes can be the candidate subject for different researches. The conventional analogue probes are required to be updated. Instead of these instruments, it is possible to develop better digital probes.

This study has not been finalized yet. The pulse oximeter telemetry system presented in this study is cheap, fast, easy to use \& setup and medical risks low example. The mechanical ventilation risks for using hypoxia control were explained. Furthermore mechanical ventilation devices are very expensive. The manual flowmeter using simple oxygen support is not enough for hypoxia control. This application eliminates both drawbacks of applications.

This study leads to some other studies such a new design of pulse oximeter that decrease the heat and other pigments effect, a new device that adjusts blood pressure, bilirubin, cholesterol, glucose levels and hemoglobin counter.

\section{References}

[1] O'Driscoll B. R., Howard L. S., Davison A. G. BTS guideline for emergency oxygen use in adult patients, Thorax, 63, vi10, 2008.

[2] Akansel N., Yıldız H. Pulse oksimetre değerlerinin güvenilir olması için neleri bilmeliyiz?, Türkiye Klinikleri Journal of Anaesthesiology \& Reanimation, 8, 44-8, 2010.

[3] Giuliano K. K., Higgins T. L. New-Generation Pulse Oximetry in the Care of Critically Ill Patients, American Journal pf Critical Care, 14, 26-39, 2005.

[4] Kelly A. M., Klim S. Agreement between arterial and venous $\mathrm{pH}$ and $\mathrm{pCO}_{2}$ in patients undergoing non-invasive ventilation in the emergency department, Emergency Medicine Journal, 25-3, 3, 2013.

[5] Hinkelbein J., Genzwuerker H.V., Sogl R., and Fiedler F. Effect of nail polish on oxygen saturation determined by pulse oximetry in critically ill patients, Resuscitation, 72, 82-91, 2007.

[6] Öncel T. U. Puls Oksimetre, Türk Yoğun Bakım Dergisi, 42, 96-106, 2006.

[7] Byrum D., Crabtree C. Ventilation Cruise control for the lungs, Nursing Made Incredibly Easy, 44-52, 2009.

[8] Cook D., Rocker G., Marshall J. Withdrawal of Mechanical Ventilation in Anticipation of Death in the Intensive Care Unit, The new england journal of medicine, 349, 1123-32,
2003.

[9] Slutsky A.S. Lung Injury Caused by Mechanical Ventilation, CHEST JOURNAL Official Publication of the American College of Chest Physicians, 116, 9-15, 1999.

[10] Esteban A., Ferguson N.D., Meade M.O., Vivar F.F., Apezteguia C. Evolution of Mechanical Ventilation in Response to Clinical Research, ATS Journals, 177, 170$177,2008$.

[11] Watthanawisuth N., Lomas T., Wisitsoraat A., Tuantranont A. Wireless wearable pulse oximeter for health monitoring using ZigBee wireless sensor network, Electrical Engineering/Electronics Computer Telecommunications and Information Technology (ECTI-CON), 2010 International Conference on, 2010.

[12] Cai Q., Sun J., Xia L., Zhao X. Implementation of a Wireless Pulse Oximeter Based on Wrist Band Sensor, Biomedical Engineering and Informatics (BMEI), 2010 3rd International Conference, 2010.

[13] Stuban N., Niwayama M. Reducing Power Consumption of Wireless Pulse Oximeters, 5th European Conference of the International Federation for Medical and Biological Engineering, 2011.

[14] Rekha Chandra R., Safeer K. P., Srividya P. Design and Development of Miniaturized Pulse Oximeter for Continuous $\mathrm{SpO}_{2}$ and $\mathrm{HR}$ Monitoring with Wireless Technology, International Journal of New Technology and Research (IJNTR), 1, 11-15, 2015.

[15] Rende A., Ayaz M., Dalkılıç G. Healthduino Mobil Sağlık İzleme Sistemi, XVII. Akademik Bilişim Konferansi AB, 2015.

[16] Adochiei F., Rotariu C., Ciobotariu R., Costin H. A Wireless Low-Power Pulse Oximetry System for Patient Telemonitoring, The 7th International Symposium on Advanced Topics In Electrical Engineering, May 12-14, 2011.

[17] Akan H., Şişman M., Taş Ü., Akbaş A. Solunum Fonksiyonlarının Gerçek Zamanlı Değerlendirilmesi İçin Gerçek Zamanlı Bir Ölçme Düzeneğinin Tasarımı, I. Uluslararası Mesleki ve Teknik Eğitim Teknolojileri Kongresi, 1174-1180, 2005.

[18] Szocik J. F., Barker S. J., Tremper K. K. Fundemental principles of monitoring instrumentation, In. Miller's Anesthesia, 1191-1226, 2005.

[19] Tremper K. K., Barker S. J. Pulse oximetry, Anesthesiology 70:98-108, 1989.

[20] Koltes- Edwards R. Pulse oximetry, In Anesthesia Secrets, 160-165, 2006.

[21] Kallistrom T.Y. Oxygen Therapy for Adults in the Acute Care Facility, AARC Clinical Practice Guideline,Respiratory Care, 47, 2002.

[22] Pologe J.A. Pulse oximetry: Technical aspects of machine design, Int Anesthesiol Clin 25:137-153, 1987. 